\title{
Three-Dimensional Electron Microscopy Simulation with the CASINO Monte Carlo Software
}

\author{
H. Demers, ${ }^{*}$ N. Poirier-Demers, ${ }^{*}$ N. de Jonge, ${ }^{* *}$ and D. Drouin*
}

* Electrical and Computer Engineering department, Universite de Sherbrooke, Sherbrooke, Quebec, J1k 2R1, Canada

** Department of Molecular Physiology and Biophysics, Vanderbilt University School of Medicine, Nashville, 37232-0615

We present a three-dimensional (3D) version of the CASINO Monte Carlo software; the current 2D version is widely used in the microscopy community. CASINO is used for the simulation of images and linescans of electron beam instruments. The software has an easy-to-use graphical user interface (GUI). The creation of the sample, setting of the simulation parameters and the viewing of the results are done through this GUI. The software now implements a full 3D sample, allowing users to create realistic geometries for their simulations. Other new features of the software include models for: 1) fast secondary and secondary electrons, 2) annular dark field scanning transmission electron microscopy (ADF STEM) [1], 3) absorbed energy, and 4) elastic cross sections based on the software ELSEPA [2] allowing modeling of the electron scattering in the range up to $500 \mathrm{keV}$. CASINO is available in 32 and 64-bit version (the latter allowing larger simulations) and uses multiCPU and multi-core hardware to reduce simulation time [3]. We will present the features of CASINO and examples of its applications.

The electron trajectory calculation is based on to the previous version of CASINO [4]. The fast secondary electrons (FSE) are generated using the Möller equation [5] while the slow secondary electrons (SE) are generated from the plasmon theory [6]. Fig. 1 shows backscattered electrons (BSE) and SE images generated with CASINO of tin balls on a carbon substrate sample. These images are used to understand the impact of microscope parameters on image resolution. The difference in contrast between the BSE and SE signal for 1 and $10 \mathrm{keV}$ incident energy is analyzed from these simulations. The largest contrast (2.3) is obtained with the SE signal at $1 \mathrm{keV}$ and is four time larger than the contrast obtained with BSE signal for the same energy.

The 3D version of the Monte Carlo software CASINO includes features to analyze the absorbed energy within the sample. These features are the simulation of complex beam scanning pattern and the calculation of the absorbed energy inside a 3D matrix unit volume. Absorbed energy modeling can assist the user in the determination of the exposure parameters and resist thickness when fabricating nanometer-scale semiconductor devices using electron beam lithography (EBL) technique. Fig. 2 shows an example of the impact of incident energy on PMMA resist lines by EBL. Fig. $2 \mathrm{~B}$ and $2 \mathrm{C}$ show a cross section view of the energy summed over $300 \mathrm{~nm}$ along the line axis in the PMMA layer. The side view at $3 \mathrm{keV}$ shows that the absorbed energy between the lines is more important at the bottom due to the larger interaction volume. From the first line at the left, we observed that the absorbed energy can occur as far as $50 \mathrm{~nm}$ (at the resist $/ \mathrm{SiO}_{2}$ interface) away from the line pattern at $3 \mathrm{keV}$. At $20 \mathrm{keV}$, no absorption was observed outside the line pattern, except for a barely visible enlargement at the bottom, which should not cause any problem during the resist development step. From this example, it is clear that such low energy would require a thinner resist layer to successfully pattern $25 \mathrm{~nm}$ dense lines. [7] 
References

[1] H. Demers et al., Microsc. Microanal., 16 (2010) 795.

[2] F. Salvat et al., Comput. Phys. Commun., 165 (2005) 157.

[3] http://www.gel.usherbrooke.ca/casino/index.html.

[4] D. Drouin et al., Scanning, 29 (2007) 92.

[5] L. Reimer, Scanning Electron Microscopy, (1998). J. R. Lowney, Scanning, 17 (1995) 281.

[6] M. Kotera et al., Jpn. J. Appl. Phys., 29 (1990) 2277. J. R. Lowney, Scanning, 18 (1996) 301.

[7] This work was supported by NIH grant R01-GM081801 (to N.P.D., H.D., and N.J.).

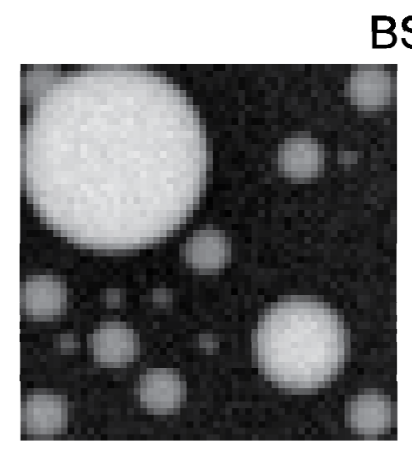

$1 \mathrm{keV}$
BSE

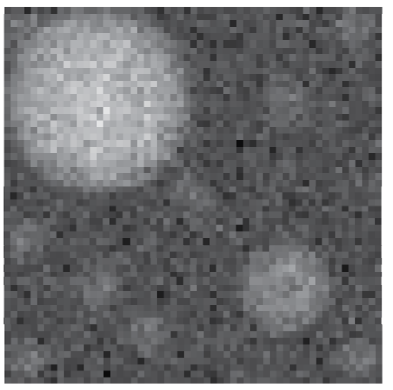

$10 \mathrm{keV}$

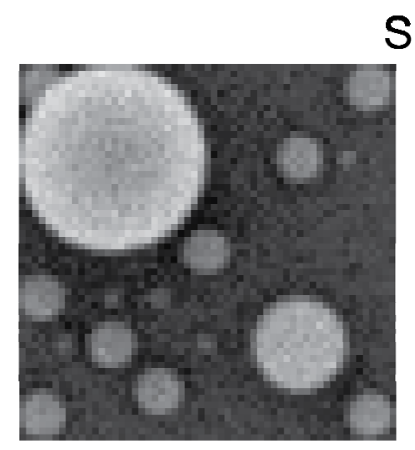

$1 \mathrm{keV}$
SE

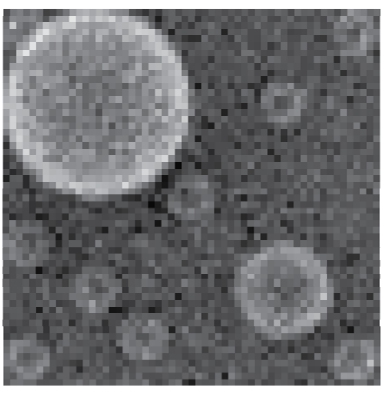

$10 \mathrm{keV}$

FIG. 1. Example of BSE and SE images at $1 \mathrm{keV}$ and $10 \mathrm{keV}$. The sample is the "standard" tin ball of various diameters $(2,5$, and $20 \mathrm{~nm})$ on carbon substrate. This example shows the difference between BSE and SE contrast and resolution depending on the electron incident energy. For each image, the field of view is $40 \mathrm{~nm} \times 40 \mathrm{~nm}$ and the grayscale intensity range is maximized to the minimum and maximum of each signal. The nominal number of electrons is 1,000 for each scan points.
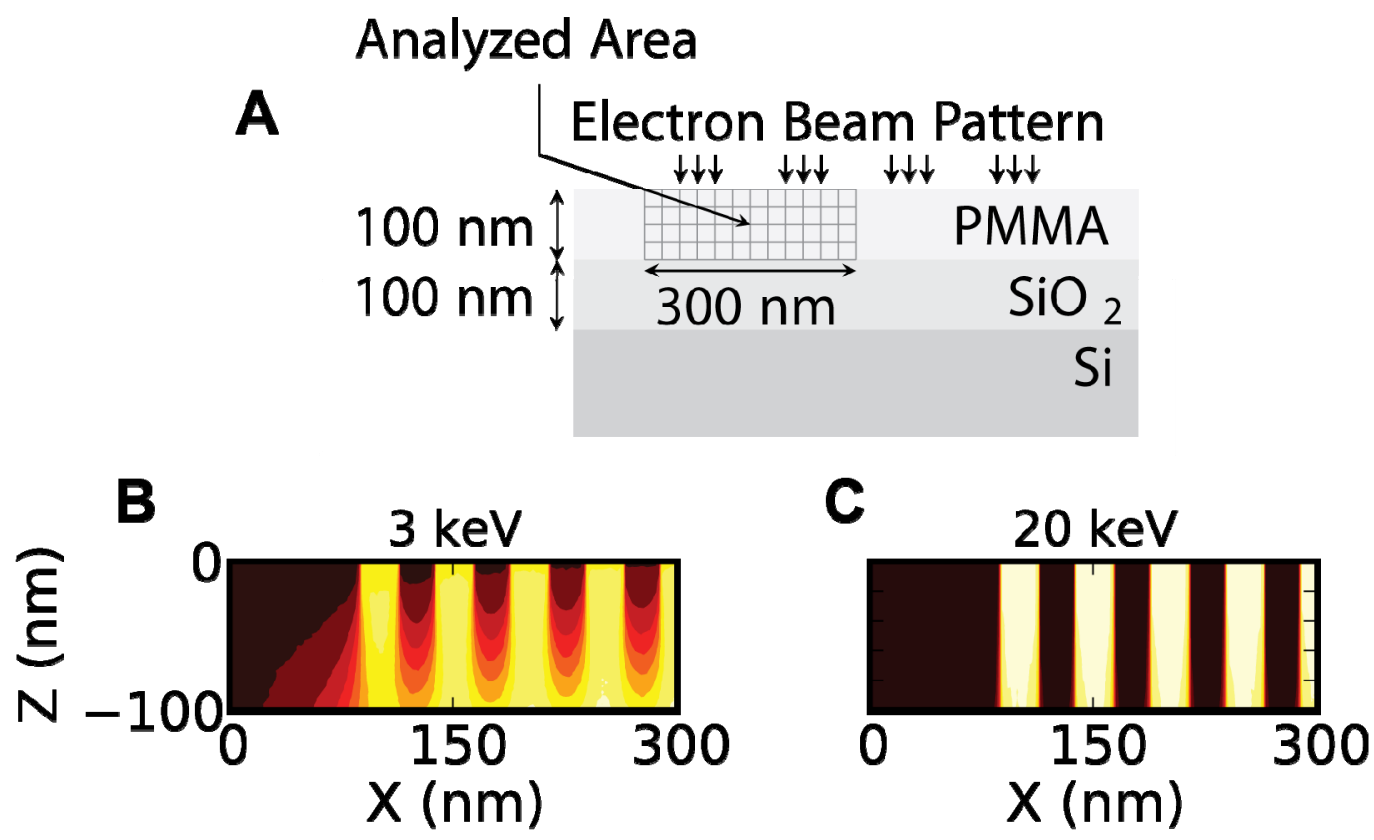

FIG. 2. Monte Carlo simulation of total absorbed energy, side view (XZ), in PMMA generated from electron beam lithography line patterns. (A) Schematic of the sample geometry (not to scale) used for the simulation. The line patterns and the analyzed area are also shown. (B) and (C) Absorbed energy image for an incident energy of 3 and $20 \mathrm{keV}$, respectively. The signal intensity is normalized with the incident energy and nominal number of electrons (300). 